\title{
Radiographic Measurement of Joint Space Width Using the Fixed Flexion View in 1,102 Knees of Japanese Patients with Osteoarthritis in Comparison with the Standing Extended View
}

\author{
Hiroyuki Kan, $\mathrm{MD}^{1,2}$, Yuji Arai, $\mathrm{MD}^{3}$, Masashi Kobayashi, $\mathrm{MD}^{4}$, Shuji Nakagawa, $\mathrm{MD}^{3}$, Hiroaki Inoue, $\mathrm{MD}^{1}$, \\ Manabu Hino, $\mathrm{MD}^{1,2}$, Shintaro Komaki, $\mathrm{MD}^{1}$, Kazuya Ikoma, $\mathrm{MD}^{1}$, Keiichiro Ueshima, $\mathrm{MD}^{1}$, \\ Hiroyoshi Fujiwara, $\mathrm{MD}^{1}$, and Toshikazu Kubo, $\mathrm{MD}^{1}$ \\ ${ }^{1}$ Departments of Orthopaedics, Graduate School of Medical Science, Kyoto Prefectural University of Medicine, Kyoto; ${ }^{2}$ Department of Orthopaedics, Kyoto \\ Interdisciplinary Institute Hospital of Community Medicine, Kyoto; ${ }^{3}$ Department of Sports and Para-Sports Medicine, Graduate School of Medical Science, Kyoto \\ Prefectural University of Medicine, Kyoto; ${ }^{4}$ Kobayashi Orthopaedic Clinic, Kyoto, Japan
}

\begin{abstract}
Purpose: The fixed flexion view (FFV) of the knee is considered useful for evaluating the joint space when assessing the severity of osteoarthritis (OA) of the knee. To clarify the usefulness of FFV for evaluation of the joint space and severity of knee OA, this study evaluated changes in the joint space on the FFV and standing extended view (SEV) in patients with knee OA.

Materials and Methods: The SEV and FFV images were acquired in 567 patients (1,102 knees) who visited the hospital with a chief complaint of knee joint pain. Medial joint space width (MJSW) and Kellgren-Lawrence (K-L) classification assessed using the SEV and FFV images were compared.

Results: Mean MJSW was significantly smaller when assessed on the FFV than on the SEV $(3.02 \pm 1.55 \mathrm{~mm}$ vs. $4.31 \pm 1.30 \mathrm{~mm}$; p<0.001). The K-L grade was the same or higher on the FFV than on the SEV.

Conclusions: The FFV is more useful than the SEV for evaluating the joint space in OA knees. Treatment strategies in patients with knee OA should be determined based on routinely acquired FFV images.
\end{abstract}

Keywords: Knee, Osteoarthritis, Radiography

\section{Introduction}

Standing plain radiography is useful for evaluating the joint space when determining the severity of osteoarthritis (OA) of

Received August 15, 2016; Revised (1st) September 26, 2016;

(2nd) October 18, 2016; Accepted October 18, 2016

Correspondence to: Yuji Arai, MD

Department of Sports and Para-Sports Medicine, Graduate School of Medical Science, Kyoto Prefectural University of Medicine, 465 Kajiicho, Kawaramachi-Hirokoji, Kamigyo-ku, Kyoto 602-8566, Japan

Tel: +81-75-251-5549, Fax: +81-75-251-5841

E-mail: yarai89046@nike.eonet.ne.jp

This is an Open Access article distributed under the terms of the Creative Commons Attribution Non-Commercial License (http://creativecommons.org/licenses/by-nc/4.0/) which permits unrestricted non-commercial use, distribution, and reproduction in any medium, provided the original work is properly cited. the knee, allowing the assessment of the grade of joint cartilage wear during routine medical practice. However, intervention by the joint cartilage and meniscus and the backward slope of the tibial joint surface can affect the standing extended view (SEV) of the knee ${ }^{1-5)}$, making accurate evaluation of the joint space difficult. To compensate, a method was designed to acquire images with a slight flexion of the knee, which enabled a more accurate evaluation of the joint space ${ }^{1)}$. More recently, simpler approaches for the acquisition of fixed flexion view (FFV) images of the knee by fixing the X-ray irradiation angle and limb position have been introduced, resulting in improved reproducibility ${ }^{6-8)}$. To date, however, there have been no large-scale studies using the FFV in Japanese patients with OA of the knee.

In FFV imaging, the anterior thigh is placed in close contact with the cassette and the tips of the toes are placed in the same 
plane as that of the cassette during acquisition. This fixes the knee flexion angle based on the physique of each patient. We hypothesized that the FFV may be more useful than the SEV for evaluating the joint space in Japanese patients. Therefore, in this study, SEV and FFV images were acquired in 567 Japanese patients (1,102 knees) diagnosed with knee OA, and SEV and FFV results were compared. To assess the value of FFV in clinical practice, we attempted to identify groups of patients for whom the FFV would be more beneficial.

\section{Materials and Methods}

\section{Subjects}

The SEV and FFV images were acquired from 567 patients (1,102 knees) who visited the hospital with a chief complaint of knee joint pain and were diagnosed with knee OA by plain radiography. The study population included 125 males (243 knees) and 442 females ( 859 knees) with a mean age of 65.4 years (range, 40 to 88 years). The SEV and FFV images were acquired for all patients, and the resulting 2,204 images were analyzed retrospectively.

\section{Radiography of the Knee Joint}

For SEV imaging, the patient stood in front of the film cassette, with the posterior thigh in direct contact with the cassette. Even if flexion contracture of the knee was present, we did not adjust for it. Irradiation was applied in the horizontal direction (Fig. 1). FFV images were acquired as described by Peterfy et al. ${ }^{6}$. Briefly, the patient stood with $10^{\circ}$ external rotation of the feet, with the anterior thigh in close contact with the cassette and the tips of the

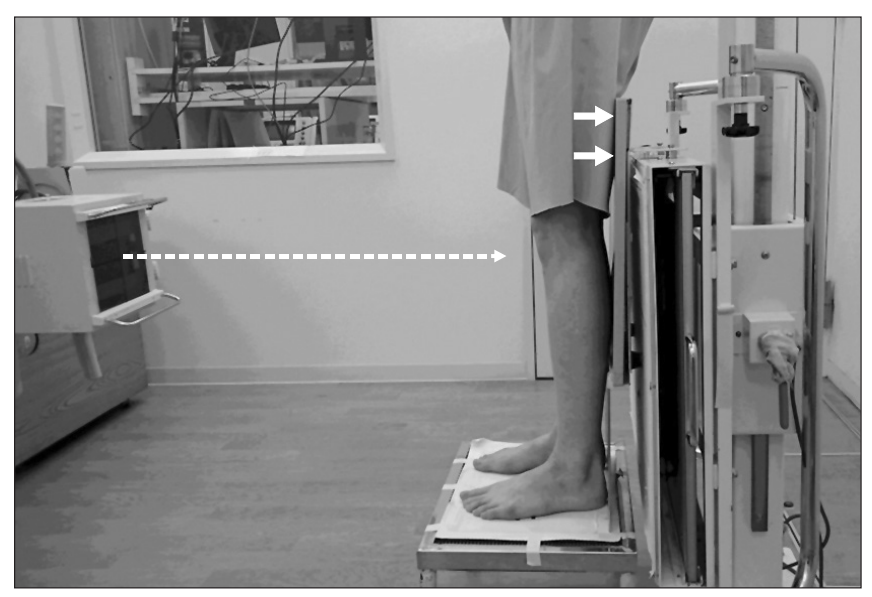

Fig. 1. The patient stood in front of the film cassette with the posterior thigh in contact with the cassette. Irradiation was applied in the horizontal direction. toes in the same plane as that of the cassette. A posteroanterior image was acquired at an irradiation angle of $10^{\circ}$ tilt in the caudal direction (Fig. 2). The acquisition conditions of SEV and FFV were the same: X-ray tube voltage, $55 \mathrm{kV}$; X-ray tube current, 100 $\mathrm{mA}$; time, $0.071 \mathrm{sec}$; source to image receptor distance, $100 \mathrm{~cm}$; and $\operatorname{grid}(-)$.

\section{Analysis}

All plain radiographs were acquired by a single radiographer, and analyzed using ApolloView Lite ver. 1.3.8.2 (Medison Acoma Nishinihon Corp., Fukuoka, Japan). Medial joint space width (MJSW) was defined as the distance between the apex of the medial condyle of the femur and the posterior end of the tibia (Fig. 3). MJSW was measured independently by two orthopedists in a

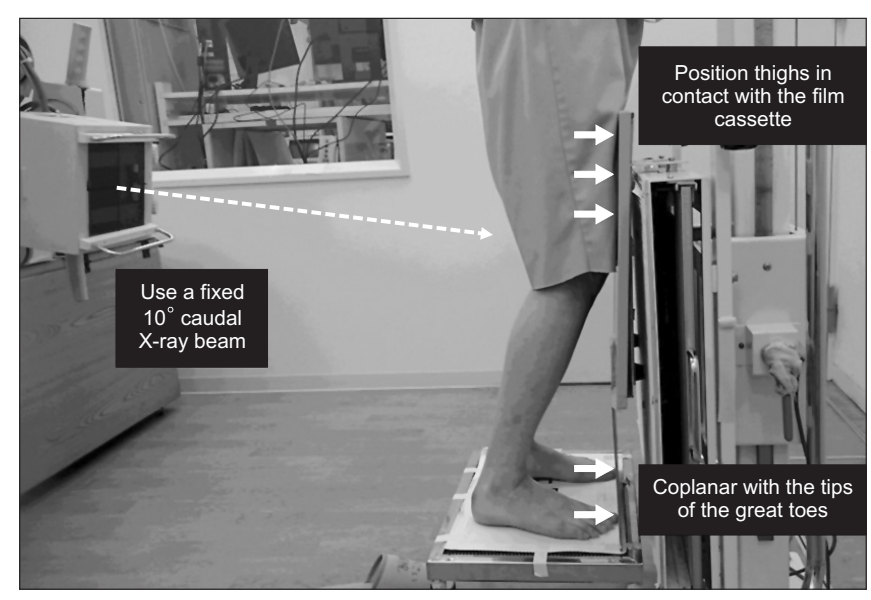

Fig. 2. The patient stood with a $10^{\circ}$ external rotation of the feet, with the anterior thigh in close contact with the cassette and the tips of the toes in the same plane as that of the cassette. A posteroanterior image was acquired at an irradiation angle of $10^{\circ}$ tilt in the caudal direction.
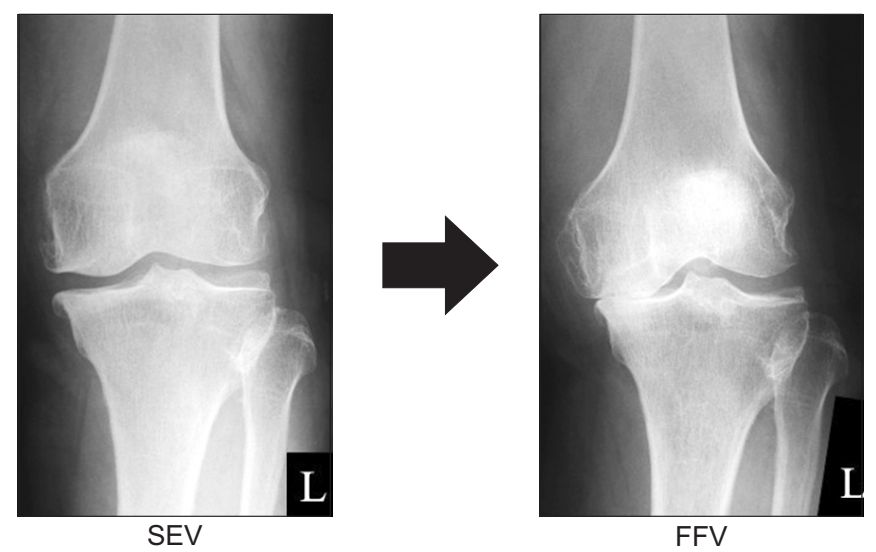

Fig. 3. Medial joint space width was defined as the distance between the apex of the medial condyle of the femur and the tibial posterior end. SEV: standing extended view, FEV: fixed flexion view, L: left. 
blinded manner, with each measurement repeated twice and averaged. Disease stage of knee OA was evaluated on X-ray images using the Kellgren-Lawrence (K-L) classification", with all images classified into four groups (grades I-IV) by severity. Inconsistent evaluations of MJSW and K-L grade by the two observers were resolved by consensus discussions.

This study was approved by the Institutional Review Board of the Kyoto Interdisciplinary Institute of Community Medicine. All subjects provided written informed consent.

\section{Statistical Analysis}

All measurements are expressed as mean \pm standard deviation. Outcomes based on the SEV and FFV were compared using paired $t$-tests. The chi-square test was used to compare the differences between the SEV and FFV. All statistical analyses were performed using IBM SPSS ver. 20.0 (IBM Co., Armonk, NY, USA), with a $\mathrm{p}<0.05$ defined statistically significant.

\section{Results}

The mean MJSW was significantly smaller on the FFV than on SEV images ( $3.02 \pm 1.55 \mathrm{~mm}$ vs. $4.31 \pm 1.30 \mathrm{~mm}$; $<<0.001)$. The $\mathrm{K}-\mathrm{L}$ grade was the same or higher based on the FFV than on the SEV. Evaluation of the severity of early stage knee OA by the SEV and FFV revealed high rates of inconsistent results (Table 1), showing statistically significant difference $(\mathrm{p}<0.001)$.

The interobserver reliability of the two orthopedists for measurement of MJSW was 0.951 . The intraobserver reliability was 0.975 for the observer 1 and 0.978 for the observer 2 .

\section{Discussion}

The most important finding of this study was that the FFV was useful when evaluating the joint space in patients with knee OA. Joint cartilage wear on the femoral side has been reported to start from a $20^{\circ}-60^{\circ}$ flexion position against the bone axis ${ }^{1)}$, and a pathological study found that cartilage destruction in knee OA occurs from the posterior knee ${ }^{10)}$. Biomechanically, maximum contact stress is generated in the femorotibial joint at $28^{\circ} \mathrm{knee}$ joint flexion ${ }^{11)}$. Furthermore, the flexion position is considered more useful than the extension position when evaluating the joint space by plain radiography because the meniscus interferes with and the tibial posterior tilt influences the evaluation ${ }^{1-8,12-14)}$. During conventional acquisition, images are acquired in a standing position, with the knee set at a flexion angle of $20^{\circ}-45^{\circ}$ using a goniometer, but it may be difficult for elderly individuals and patients with marked arthralgia to retain this posture ${ }^{1,2,8,12,13,15)}$. In acquiring FFV images, the anterior thigh is placed in close contact with the cassette and the tips of the toes are placed in the same plane as that of the cassette, thus fixing the knee flexion angle according to the physique of each patient ${ }^{6}$. Although variability of knee flexion angle might affect the evaluation, this makes retention of the limb position easier and enables stable acquisition, as well as reducing errors with regard to the knee flexion angle and the distance from the cassette ${ }^{8,16)}$. The Lyon Schuss view (LSV), which uses the same posture as the FFV to acquire images, is also considered useful for joint space evaluation, as well as being more reproducible than the $\mathrm{SEV}^{3,17}$. In the FFV, the irradiation angle is fixed in a $10^{\circ}$ caudal direction, based on fluoroscopy results. In contrast, the LSV requires fluoroscopic adjustment of the irradiation angle to the medial plateau of the tibia. The LSV is more accurate for measuring actual joint space width than the $\mathrm{FFV}^{18)}$, but it increases the exposure dose and makes the procedure more complex and time-consuming.

An extensive literature search revealed no reports of anatomical features of Japanese knees. But Yue et al. ${ }^{19)}$ reported the anatomical features of Chinese knees that we presume to be of the same Asian type. They reported that there was no difference in the medial/lateral posterior slope between Chinese and white subjects in either sex. Thus, to assess the usefulness of FFV in evaluating the severity of knee OA in Japanese patients, we compared the FFV and SEV images. Overall, MJSW was significantly smaller on the

Table 1. Comparison of Kellgren-Lawrence (K-L) Grades in Knees Evaluated by Standing Extended View and Fixed Flexion View Imaging

\begin{tabular}{cccccc}
\hline \multirow{2}{*}{$\begin{array}{c}\text { Standing extended view } \\
\text { (K-L grade) }\end{array}$} & I & II & Fixed flexion view (K-L grade) & IV & Total \\
\hline I & $374(76)$ & $20(4)$ & $93(19)$ & $6(1)$ & 493 \\
II & 0 & $151(39)$ & $173(45)$ & $54(16)$ & 388 \\
III & 0 & 0 & 0 & $110(67)$ & $5(100)$ \\
IV & 0 & 0 & 321 & 236 & 56 \\
Total & 374 & 171 & 1,102 & 55 \\
\hline
\end{tabular}

Values are presented as number (\%). 


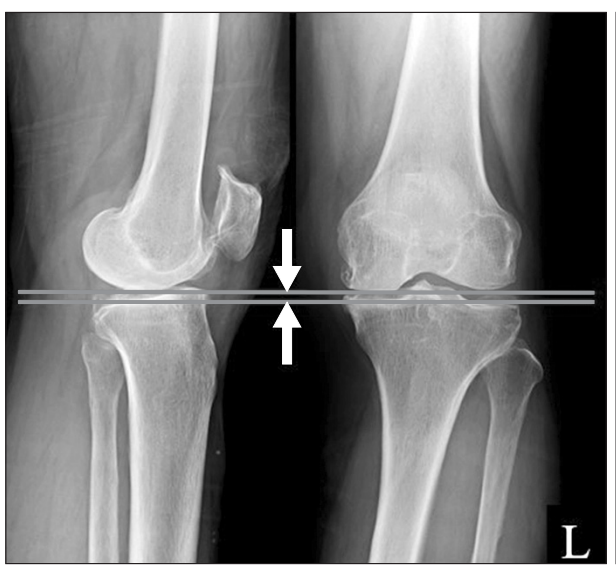

SEV

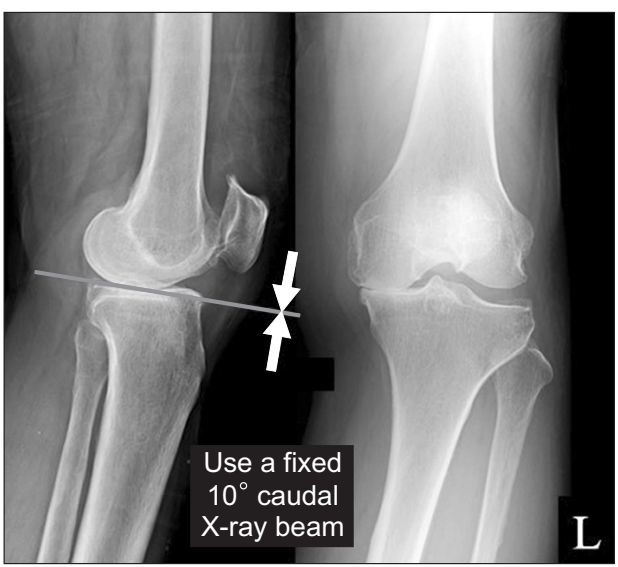

FFV
Fig. 4. The Medial joint space width values were smaller on the fixed flexion view (FFV) than on the standing extended view (SEV) for all patients. L: left.
FFV than on the SEV images, and the K-L grade was the same or higher on the FFV than on the SEV as observed in other populations ${ }^{7)}$. Therefore, the FFV was more useful than the SEV in evaluating the severity of knee OA in Japanese patients in routine medical practice.

In the present study, wear of the cartilage was in progress, regardless of the remaining cartilage evaluated by the SEV. Evaluation of the severity of early stage knee OA based on the SEV alone has been reported insufficient ${ }^{14)}$. Evaluation of the severity of early stage knee OA by the SEV and FFV resulted in high rates of inconsistent results, suggesting that the SEV underestimates the OA severity, particularly during early stages (Fig. 4). In contrast, another study reported a higher concordance rate of K-L grade assessed on the SEV and $\mathrm{FFV}^{7)}$ : of the knees classified as $\mathrm{K}$-L grade I on the SEV, 92\% were classified as grade I and $8 \%$ as a higher grade on the FFV; of the knees classified as K-L grade II on the SEV, $85 \%$ were classified as grade II and $15 \%$ as a higher grade on the FFV; and of the knees classified as K-L grade III on the SEV, $96 \%$ were classified as grade III and $4 \%$ as a higher grade on the FFV. Differences between study results may have been affected by differences in the subject age, number of knees, measuring method, and physique.

The results of the present study suggest that FFV images be acquired routinely in patients who visit the hospital with a chief complaint of knee joint pain. Treatment strategies for knee OA require accurate evaluations of the severity and grade of progression of knee OA in addition to clinical symptoms. Loss of MJSW may require high tibial osteotomy ${ }^{20-22)}$, unicompartmental knee arthroplasty, or total knee arthroplasty ${ }^{23)}$. However, when residual MJSW is present, the knee can be treated with a joint conserving surgery, including osteotomy. Determining the optimal surgical procedure requires accurate evaluation of the joint space on FFV images.

\section{Limitations}

There are several limitations of this study. First, knee extension and flexion views show different parts of the knee joint cartilage status. Joint cartilage wear on the femoral side has been reported to start from a $20^{\circ}-60^{\circ}$ flexion position against the bone axis ${ }^{1)}$. We were able to evaluate the site where cartilage was more reduced on the FFV than on the SEV. However, these radiographic views could not reflect any superiority of either method in expressing the OA grade. Thus, a long-term radiographic followup is needed to determine any superiority of either method in expressing the OA grade. Second, strictly speaking, analyzing the joint space in $\mathrm{mm}$ was not possible since the radiographs were not calibrated. In the case with flexion contracture, the magnification percentage of the radiograph could have been larger than that in the case without flexion contracture. However, the FFV was found to be useful for evaluating the joint space in OA knees in daily medical practice.

\section{Conclusions}

MJSW was significantly smaller on the FFV than on the SEV, whereas the K-L grade was the same or higher on the FFV than on the SEV. The FFV can be more useful than the SEV for evaluating the joint space in OA knees. Treatment strategies for knee OA should include the routine acquisition of FFV images in patients with a chief complaint of knee joint pain.

\section{Conflict of Interest}

No potential conflict of interest relevant to this article was reported. 


\section{References}

1. Rosenberg TD, Paulos LE, Parker RD, Coward DB, Scott SM. The forty-five-degree posteroanterior flexion weight-bearing radiograph of the knee. J Bone Joint Surg Am. 1988;70:147983.

2. Deep K, Norris M, Smart C, Senior C. Radiographic measurement of joint space height in non-osteoarthritic tibiofemoral joints: a comparison of weight-bearing extension and 30 degrees flexion views. J Bone Joint Surg Br. 2003;85: 980-2.

3. Vignon E, Piperno M, Le Graverand MP, Mazzuca SA, Brandt KD, Mathieu P, Favret H, Vignon M, Merle-Vincent F, Conrozier T. Measurement of radiographic joint space width in the tibiofemoral compartment of the osteoarthritic knee: comparison of standing anteroposterior and Lyon schuss views. Arthritis Rheum. 2003;48:378-84.

4. Hunter DJ, Buck R, Vignon E, Eckstein F, Brandt K, Mazzuca SA, Wyman BT, Otterness I, Hellio Le Graverand MP. Relation of regional articular cartilage morphometry and meniscal position by MRI to joint space width in knee radiographs. Osteoarthritis Cartilage. 2009;17:1170-6.

5. Hellio Le Graverand MP, Buck RJ, Wyman BT, Vignon E, Mazzuca SA, Brandt KD, Piperno M, Charles HC, Hudelmaier M, Hunter DJ, Jackson C, Kraus VB, Link TM, Majumdar S, Prasad PV, Schnitzer TJ, Vaz A, Wirth W, Eckstein F. Subregional femorotibial cartilage morphology in women: comparison between healthy controls and participants with different grades of radiographic knee osteoarthritis. Osteoarthritis Cartilage. 2009;17:1177-85.

6. Peterfy C, Li J, Zaim S, Duryea J, Lynch J, Miaux Y, Yu W, Genant HK. Comparison of fixed-flexion positioning with fluoroscopic semi-flexed positioning for quantifying radiographic joint-space width in the knee: test-retest reproducibility. Skeletal Radiol. 2003;32:128-32.

7. Niinimaki T, Ojala R, Niinimaki J, Leppilahti J. The standing fixed flexion view detects narrowing of the joint space better than the standing extended view in patients with moderate osteoarthritis of the knee. Acta Orthop. 2010;81:344-6.

8. Buckland-Wright C. Review of the anatomical and radiological differences between fluoroscopic and non-fluoroscopic positioning of osteoarthritic knees. Osteoarthritis Cartilage. 2006;14 Suppl A:A19-31.

9. Kellgren JH, Lawrence JS. Radiological assessment of osteoarthrosis. Ann Rheum Dis. 1957;16:494-502.

10. Messieh SS, Fowler PJ, Munro T. Anteroposterior radio- graphs of the osteoarthritic knee. J Bone Joint Surg Br. 1990; 72:639-40.

11. Maquet PGJ. Biomechanics of the knee: with application to the pathogenesis and the surgical treatment of osteoarthritis. New York: Springer-Verlag; 1976. p1-237.

12. Davies AP, Calder DA, Marshall T, Glasgow MM. Plain radiography in the degenerate knee. A case for change. J Bone Joint Surg Br. 1999;81:632-5.

13. Inoue $S$, Nagamine $R$, Miura $H$, Urabe K, Matsuda $S$, Sakaki $\mathrm{K}$, Iwamoto Y. Anteroposterior weight-bearing radiography of the knee with both knees in semiflexion, using new equipment. J Orthop Sci. 2001;6:475-80.

14. Merle-Vincent F, Vignon E, Brandt K, Piperno M, CouryLucas F, Conrozier T, Mathieu P, Hellio Le Graverand MP. Superiority of the Lyon schuss view over the standing anteroposterior view for detecting joint space narrowing, especially in the lateral tibiofemoral compartment, in early knee osteoarthritis. Ann Rheum Dis. 2007;66:747-53.

15. Duddy J, Kirwan JR, Szebenyi B, Clarke S, Granell R, Volkov S. A comparison of the semiflexed (MTP) view with the standing extended view (SEV) in the radiographic assessment of knee osteoarthritis in a busy routine X-ray department. Rheumatology (Oxford). 2005;44:349-51.

16. Nevitt MC, Peterfy C, Guermazi A, Felson DT, Duryea J, Woodworth T, Chen H, Kwoh K, Harris TB. Longitudinal performance evaluation and validation of fixed-flexion radiography of the knee for detection of joint space loss. Arthritis Rheum. 2007;56:1512-20.

17. Piperno M, Hellio Le Graverand MP, Conrozier T, Bochu M, Mathieu P, Vignon E. Quantitative evaluation of joint space width in femorotibial osteoarthritis: comparison of three radiographic views. Osteoarthritis Cartilage. 1998;6:252-9.

18. Mazzuca SA, Hellio Le Graverand MP, Vignon E, Hunter DJ, Jackson CG, Kraus VB, Link TM, Schnitzer TJ, Vaz A, Charles HC. Performance of a non-fluoroscopically assisted substitute for the Lyon schuss knee radiograph: quality and reproducibility of positioning and sensitivity to joint space narrowing in osteoarthritic knees. Osteoarthritis Cartilage. 2008;16:1555-9.

19. Yue B, Varadarajan KM, Ai S, Tang T, Rubash HE, Li G. Differences of knee anthropometry between Chinese and white men and women. J Arthroplasty. 2011;26:124-30.

20. Lee DC, Byun SJ. High tibial osteotomy. Knee Surg Relat Res. 2012;24:61-9.

21. Kim HJ, Yoon JR, Choi GW, Yang JH. Imageless navigation versus conventional open wedge high tibial osteotomy: a 
68 Kan et al. Fixed Flexion View vs. Standing Extended View for Evaluation of OA Severity

meta-analysis of comparative studies. Knee Surg Relat Res. 2016;28:16-26.

22. Akizuki S, Shibakawa A, Takizawa T, Yamazaki I, Horiuchi H. The long-term outcome of high tibial osteotomy: a ten- to 20-year follow-up. J Bone Joint Surg Br. 2008;90:592-6.
23. Pandit H, Jenkins C, Barker K, Dodd CA, Murray DW. The Oxford medial unicompartmental knee replacement using a minimally-invasive approach. J Bone Joint Surg Br. 2006;88: 54-60. 